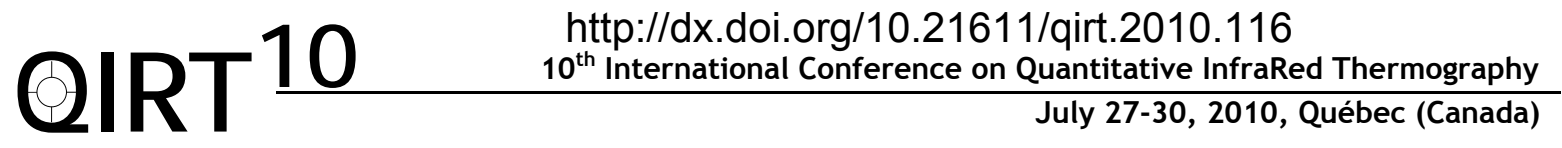

\section{Application of thermal camera to optimize assembly methods of laser diodes}

\author{
by J. Rybiński ${ }^{1}$, M. Bednarek ${ }^{1}$, P. Wiśniewski ${ }^{2}$, T. Świetlik ${ }^{2}$ \\ ${ }^{1}$ The Main School of Fire Service, 52/54 Słowackiego St., 01-629 Warsaw, Poland \\ ${ }^{2}$ Institute of High Pressure Physics of the Polish Academy of Sciences (PAN), 29/37 Sokołowska St., \\ 01-142 Warsaw, Poland
}

Corresponding author: j.rybinski@sgsp.edu.pl

Keywords: microscope thermography, blue lasers, semiconductor lasers

\section{Introduction}

The majority of electronic devices exert considerable amounts of heat during operation, and its effective disposal gains particular importance as the excessive temperature of semiconductor devices has a clearly negative impact on their operation. First of all, properties of semiconductor materials frequently depend to a large extent on temperature, and so temperature changes are a cause of a lack of stability of parameters of semiconductor devices. Furthermore, degradation processes of semiconductor materials are frequently thermally activated and occur much quicker at increased temperature, consequently shortening the lifetime of the devices involved. An additional mechanism which accelerates the degradation of electronic devices is the mechanical stress that occurs for materials having different temperature expansion coefficients, which is of particular importance during switching them on and off. This problem tends to gain importance, because with progress in miniaturization in electronics, the density of generated power grows. For optimizing the method of heat removal the knowledge of temperature distribution during normal device operation is crucial.

The subject of this study were the determination of temperature distribution in violet light emitting, nitride-based laser diodes.

Laser semiconductor diodes belong to devices characterized by the highest densities of generated power. The reason for this is that condition that allows lasing is achievement of a high current density in the active area of a laser diode. To meet this requirement the active area is designed in the form of a narrow strip of a width ranging from single microns for lasers of a lower power, up to a few tens of microns for higher power lasers [1][2]. As regards lasers produced on the basis of nitrides, power generated in the laser structure is even higher than in the case of red and infrared lasers, because owing to specific properties of those semiconductor materials, laser operation requires higher current density (3-10 kA/ $\left.\mathrm{cm}^{2}\right)$ and voltage (over $4 \mathrm{~V}$ ), which leads to a very high density of electric power (12$50 \mathrm{~kW} / \mathrm{cm}^{2}[3][4]$.

To achieve effective continuous wave laser operation it is indispensable to obtain appropriately low thermal resistance of the entire device. Determination of the active laser area temperature can be achieved by using various optical methods: electroluminescence, Raman spectroscopy, thermoreflection, or electrical methods, such as testing the voltage of the p-n junction. However, the above mentioned methods do not supply information on temperature distribution outside the active area of the laser and in its housing, which is necessary to allow optimization the heat dissipation. This type of data may be directly obtained from microscopic thermography.

\section{Measurements}

The measurements have been carried out on a stand comprising a thermovision camera Raytheon Radiance HSX with a germanium made lens for macroscopic testing with maximum spatial definition $8 \mu \mathrm{m}$. It is a radiometric camera with a cooled detector of InSb, working within the operational spectral range of 3 - $5 \mu \mathrm{m}$. 
The performed experiments were aimed at determining temperature distribution in a semiconductor laser, mounted in two different configurations: a standard $5.6 \mathrm{~mm}$ laser housing (TO18) and a special, custom made two copper blocks laser mount. In both cases laser was soldered with AuSn to the diamond heatspreader with the active side faced at a diamond (p-type down). The objective was to detect places with the highest thermal resistance. This was to allow determination of the effectiveness of heat removal from the laser active area and proposing methods for further assembly optimization and as a consequence of reducing the threshold current and to slow down laser degradation.

To verify test results and the applied temperature calibration further temperature measurements were carried out based on a comparison of current-voltage characteristics at impulse and continuous power supply. The differences in voltage allowed the determination of temperature on the junction at the given supply current to the maximum value of $100 \mathrm{~mA}$, at which pulse power supply does not cause structure heating yet. The measurements of the temperature of the junction for low supply current performed by studying its electrical properties well match the results obtained from thermovision measurements for supply current of 200-400 mA, at which typically the laser action was initiated.

\section{Conclusions}

The application of thermovision camera for visualization and measurement of temperature distribution in laser diode device enabled the following conclusions:

- the use of a heatspreader is necessary for continuous wave operating lasers,

- the following material junctions: laser/heatspreader and heatspreader/heatsink are the most important and need to be optimized

- $\quad$ in the case of standard $5.6 \mathrm{~mm}$ laser diode housing very good thermal coupling to the heatsink is of importance and also needs optimisation.

The conducted tests have shown that the thermovision camera has a potential in characterizing microelectronic devices like semiconductor laser diodes and can be considered as a complementary tool in establishing thermal characteristics of these devices.

\section{REFERENCES}

[1] A. Coldren, S. W. Corzine, Diode lasers and Photonic Integrated Circuits, John Wiley \& Sons, Inc., New York 1995.

[2] R. Diehl (ed.), High-Power Lasers, Springer-Verlag, Berlin Heidelberg 2000.

[3].H. Morkoç, Nitride Semiconductors and Devices,Springer-Verlag, Berlin Heidelberg 1999;

[4] P. Perlin, M. Leszczyński, P. Prystawko, M., Boćkowski, I. Grzegory, C. Skierbiszewski, T. Suski „Laser diodes grown on gallium nitride substrates”,III- Nitride Devices and NanoEngineering, editor Zhe Chuan Feng, pp 223-252, Imperial College Press, London 2008. 